\title{
Monitoring of earthen long linear embankments by geophysical tools integrated with geotechnical probes
}

\author{
Stefano Utili, ${ }^{1 *}$ \\ ${ }^{1}$ School of Engineering, Newcastle University, Newcastle upon Tyne, NE1 7RU, United Kingdom
}

\begin{abstract}
The use of electrical conductivity measurements from a non-invasive hand held electromagnetic probe is showcased to monitor the water content of earthen embankments at routine inspections. A methodology to convert the electrical conductivity measurements from the electromagnetic device into water content values is illustrated. The methodology is based on measuring the soil electrical conductivity variation with respect to a baseline reference condition and calibrating a water content - electrical conductivity relationship by comparing electrical conductivity readings from the electromagnetic probes with water content readings taken from geotechnical probes installed in a few sections of the embankment. The values of water content converted from the conductivity measurements according to the proposed procedure were found to be in very good agreement with independent measures of water content taken at times well beyond the calibration period.
\end{abstract}

\section{Introduction}

Earthen embankments are built for a variety of purposes the main ones being transportation, e.g. road and railway embankments, and to act as flood barriers. Usually flood defence embankments are made of lower quality materials than transportation embankments [1]. With regard to the latter, the increased focus on sustainability in the engineering sector brings renewed attention to health monitoring in the long term; while with regard to the former, they are subject to significant deterioration due to aging [2] so increasingly large resources are dedicated to their health monitoring.

Monitoring and condition assessment of embankments worldwide are mainly carried out by visual inspections at set intervals [3-5]. Purely visual inspections present several shortcomings, namely inconsistencies due to different levels of training and experience of the inspectors and providing qualitative measurements of deterioration rather than quantitative ones with failures often occurring without any visible warning signs of deterioration being detected. Therefore, experts agree on the fact that although visual inspection provides extremely valuable information, inspections alone cannot be relied upon to assess the fitness of earthen embankments [3, 6].

Here, the use of non-invasive electromagnetic geophysical probes is advocated for the long term monitoring of the water content in embankments made of cohesive soils. The probe employed in the study is a hand held devise that can be easily operated by untrained personnel and that could be added to the standard inspector kit. Potentially it could also be used to monitor material deterioration occurring over long time spans. In the following it will be shown how periodic measurements of electrical conductivity taken by a portable electromagnetic devise can be converted into water content. A methodology to calibrate the electrical conductivity - water content relationship was first proposed in [7]. In this paper a new simplified methodology is proposed with the aim of extending the application of the method to cases of embankments where less data are available, e.g. absence of weather stations and few geotechnical probes.

\section{Measurements}

In 2007, the construction of an earthen flood defence embankment enclosing a floodplain along the river Irvine was completed in Galston (Scotland, UK). The embankment is made of an uppermost layer $(5-10 \mathrm{~cm})$ of a sandy topsoil below which lies a core of glacial till containing several boulders (Figure 1).

\subsection{Electromagnetic probe}

Several electromagnetic probes measure the electrical conductivity of the ground. The CMD-2 probe from Gf Instruments was chosen since it is cheap and simple to use. In [8] the working principles of the device are illustrated. Measurements were taken by an operator walking on the horizontal upper surface (crest) of the embankment along the longitudinal direction at a constant pace of $5 \mathrm{~km} / \mathrm{h}$, holding the CMD-2

Corresponding author: author@e-mail.org 
approximately $1 \mathrm{~m}$ above ground with the device oriented perpendicular to the longitudinal direction.

\subsection{Geotechnical suite}

To calibrate the electrical conductivity - water content relationship, measurements of water content were taken by a geotechnical suite over a period of two years into two cross-sections (A and B in Figure 1a) with the property of being perpendicular to each other to better account for the influence of topographical orientation on water content.

Two different instruments were employed: a portable profile probe (PR2, Delta-T Devices) to measure water content along several vertical lines up to a depth of $1 \mathrm{~m}$, and a portable diviner (Diviner 2000, Sentek, Stepney Australia) to measure water content along various vertical lines every $10 \mathrm{~cm}$ from ground level down to 1.6 $\mathrm{m}$ of depth. In section $\mathrm{A}, 10$ access tubes were employed: 5 for the profile probe and 5 for the diviner. In section $B$ there were 12 measuring points: 6 for the profile probe and 6 for the diviner. Both tools were carefully calibrated in situ (for details see [7]).

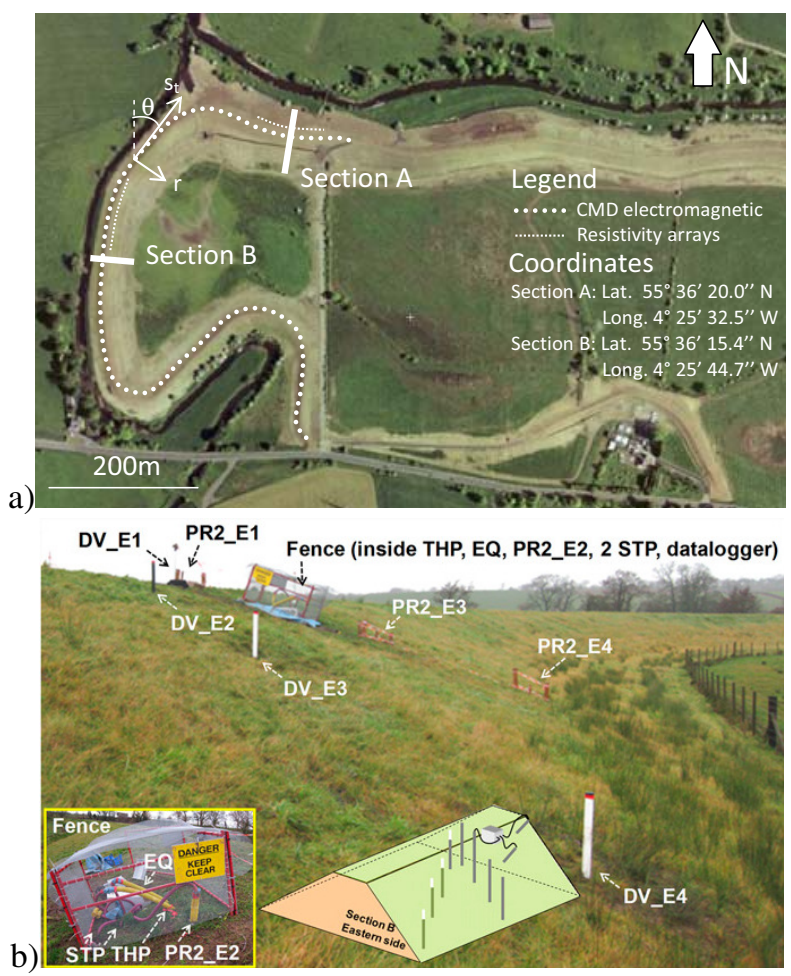

Fig. 1. a) plan view of the monitored embankment; b) crosssection B with probes to measure water content (after [7]).

\section{Calibration of the geotechnical suite}

Regarding the calibration exercise undertaken, only the glacial till is of interest since the thickness of the top soil in the embankment flanks, where all the measurements were taken, is $5 \mathrm{~cm}$ and the measurements were taken at depths always larger than $10 \mathrm{~cm}$. A sequential approach was adopted.
The calibration curve for the theta probe (THP) was taken from [9] who calibrated the THP using samples of till retrieved from the same quarry (Hallyards, Scotland, UK) from which the till of our monitored embankment was extracted. The till was retrieved from the quarry at five different known water contents and compacted into five cylindrical containers with the same compaction effort as in the monitored embankment, i.e. relative compaction of $95 \%$ with compaction control performed according to the Specification for Highway Works: Earthworks, Series 6000 (Highways Agency, 2006). Given the high level of compaction and the non-swelling nature of this glacial till, the variations of dry unit weight in the embankment over time and space occurring for the range of measured in-situ water contents can be considered negligible (less than $+/-5 \%$ ). Hence, we transformed the volumetric water contents measured by the geotechnical probes (profile probe, diviner, etc.) into gravimetric water contents assuming the dry unit weight of the till at its optimal water content, i.e. a maximum dry unit weight of $19.1 \mathrm{kN} / \mathrm{m} 3$. This value was obtained as average of 7 measurements performed in the laboratory on undisturbed U-38 samples retrieved from the embankment. This value turned out to be almost identical to the value measured by [10] on a small scale embankment built from the same glacial till reconstituted in the laboratory.

The calibration of the profile probe (PR) was obtained by comparing the raw data in millivolts obtained by the PR located at the gate in section B (PR2_E2 in Figure 1b) at 20 and $30 \mathrm{~cm}$ depth with the measurements of the theta probe (THP) in the same location (20 cm longitudinally away from the PR2_E2 in Figure $1 \mathrm{~b}$ ) at $25 \mathrm{~cm}$ depth for a 4 month period. Data were recorded every 4 hours. A strong similarity between the trends of the readings of the two probes plotted in Figure 2 can be observed. Since the two devices measure the water content of the same portion of soil, a calibration procedure, based on the visual match of the curves, was adopted: the scale of the ordinate axis of the THP readings (expressed in $\mathrm{mV}$ ) was varied until it satisfactorily matched the average (not reported in the figure to avoid cluttering) of the two measurements at 20 and $30 \mathrm{~cm}$ depth obtained by the THP. The resulting linear relationship between the values measured by the PR and the values measured by the THP is shown in Figure 2. This relationship was used to convert the readings of the $\mathrm{PR}$ into equivalent milliVolt units measured by the THP, and then they were in turn transformed into gravimetric water content employing the calibration curve of the THP.

Following the same approach, indirect calibration of the diviner (D) was performed by varying the scale of the frequencies measured by the $\mathrm{D}$ until obtain a satisfactory match with the water content profile measured by the PR in the corresponding access tube. The corresponding PR access tube is the one aligned with the D tube in the longitudinal direction (e.g. DV_S3 and PR2_S3, see Figure 1b). In Fig. 3a, two of the performed calibrations are shown. The curve shown in Fig. $3 \mathrm{~b}$ was obtained by repeating this procedure for all the monitoring points of the $\mathrm{D}$ in the period considered. 


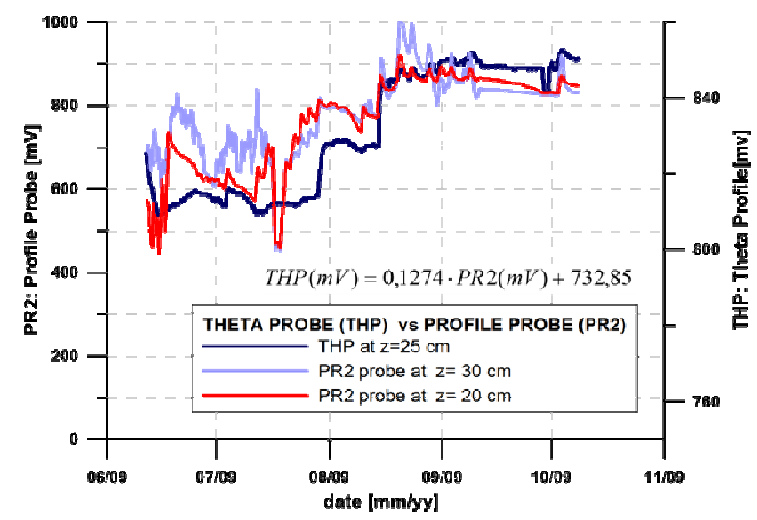

Fig. 2. (a) Calibration of the profile probe: measurements by the theta probe and the profile probe taken at the same location, PR2_E2 (see Figure 1).

Since the calibration procedures adopted for the PR and the $\mathrm{D}$ are based on cross-correlation, errors of measurement could be amplified. Hence, in order to check the amount of error amplification, direct measurements of the gravimetric water content were obtained via small in-situ samples taken in several points of the embankment on the same day (see the triangles in Fig. 3a).

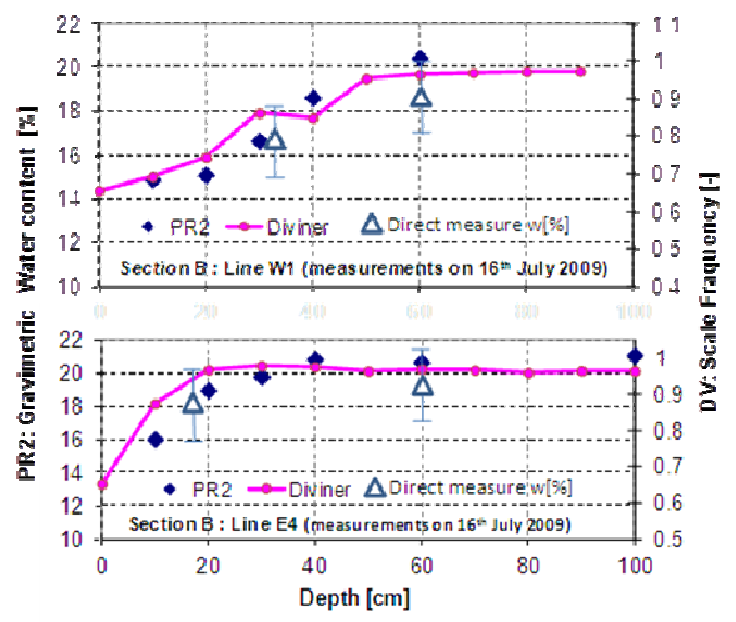

a)

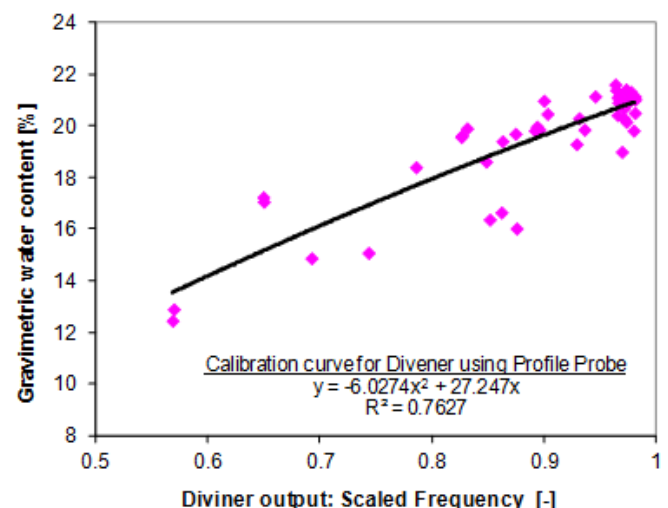

b)

Fig. 3. (a) Calibration of the diviner by comparison with the measurements of the profile probe: a) examples of cross comparisons for measurements taken in access tubes lying along the longitudinal line $\mathrm{W} 1$ and E4. The triangles with their error bar indicate the values of water content obtained via direct measurement. b) Obtained calibration curve for the diviner.
Looking at Fig. 3a, a good agreement between the values of water content measured by the indirectly calibrated probes (PR and D) and direct measurements emerges so that it can be concluded that error propagation is within acceptable limits.

Overall, the adopted calibration procedure has the obvious advantage of requiring only small samples of soil for the direct calibration of the THP in the laboratory with the PR and D indirectly calibrated in-situ. Conversely, direct calibration of the PR and D would require retrieving large volumes of soil, to avoid the influence of boundary effects, at predefined water contents to the laboratory.

\section{Establishment of the water content function}

Herein the variable $\sigma$ is employed to represent the ground electrical conductivity which is a function of both space and time, hence $\sigma=\sigma(x, s, z, t)$. Electromagnetic probes only provide a measure of $\sigma$ which is averaged over a prismatic volume of ground where the induced electrical field is non-zero. Considering a generic cross-section of the embankment, we define:

$$
\bar{\sigma}(s, t)=\frac{\int_{A_{C M D}} w(x, s, z, t) d x d z}{A_{C M D}}
$$

with $\mathrm{A}_{\mathrm{CMD}}=b^{*} d, \mathrm{~b}=$ the distance between the two ends of the electromagnetic probe (hence corresponding to the width of the portion of the embankment cross-section where the induced electrical field is non-zero) and $d=$ so-called effective depth, i.e. the depth of the induced electromagnetic field. Note that $\mathrm{A}_{\mathrm{CMD}}$ is independent of the cross-section considered. The effective depth is a function of the type of ground and of the vertical distance from the portable device to ground level. $d$ is an unknown to be determined by selecting the value providing the best correlation between measurements of electrical conductivity and water content (see Figure 4a).

$$
\overline{\bar{\sigma}}(t)=\frac{\int_{s=0}^{s=L} \bar{\sigma}(s, t) d s}{L}
$$

with the second above score bar denoting spatial average over the longitudinal coordinate $s$. Then, we can introduce the normalised cross-sectional average electrical conductivity as:

$$
\bar{\sigma}_{0}(s, t)=\frac{\bar{\sigma}(s, t)}{\overline{\bar{\sigma}}(t)}
$$


The normalised measurements taken at $t_{k}$, i.e. $\bar{\sigma}_{0_{k}}=\bar{\sigma}_{0}\left(s, t=t_{k}\right)$, are plotted in Figure $4 \mathrm{~b}$. From the figure, it emerges that the curves coincide almost perfectly. This leads to consider the average of $\bar{\sigma}_{0_{k}}$ over time:

$$
\overline{\bar{\sigma}}_{0}(s)=\underset{k}{\operatorname{average}} \bar{\sigma}_{0}\left(s, t=t_{k}\right)=\underset{k}{\operatorname{average}}\left(\frac{\bar{\sigma}\left(s, t=t_{k}\right)}{\overline{\bar{\sigma}}\left(t=t_{k}\right)}\right)
$$

as the representative curve of the conductivity of the embankment with the underscore bar denoting time average.

a)
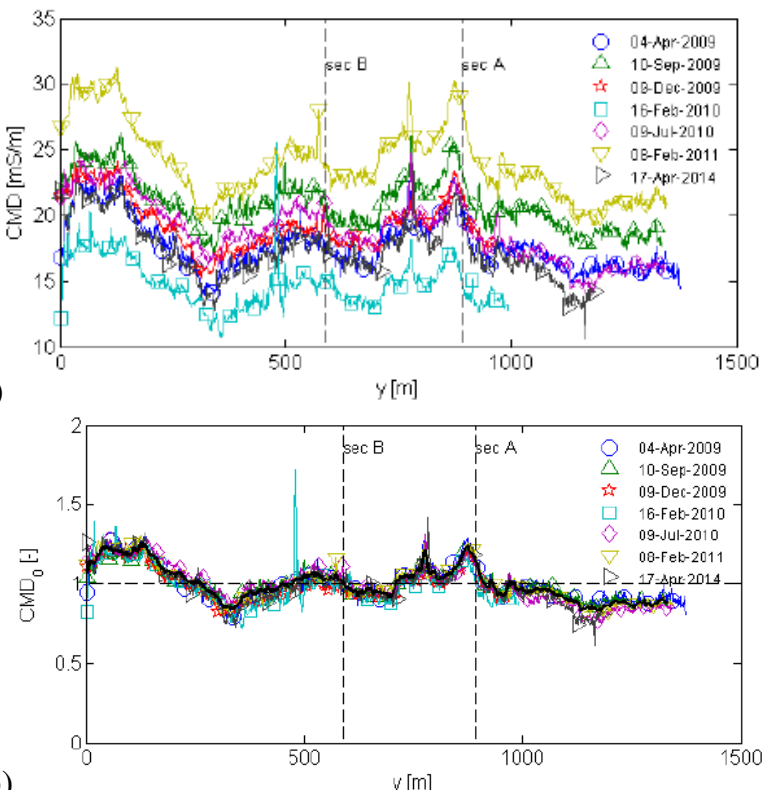

b)

$y[\mathrm{~m}]$

Fig. 4. (a) electrical conductivity measurements along the embankments at seven different times; (b) normalised values of conductivity measurements (after [7]).

The time-independent function $\bar{\sigma}_{0}(s)$ can be thought of as a unique identifier of the embankment expressing the variation of conductivity along the $\mathrm{s}$ coordinate due to the variation of geometrical, hydraulic and lithological properties and to the effects of exposure to weather conditions. On the other hand, the function $\overline{\bar{\sigma}}(t)$ reflects the temporal effect of climatic variations (e.g. rainfall, wind, temperature variations, etc.) and aging on the ground electrical conductivity.

Analogously to $\bar{\sigma}(s, t)$, we define $\bar{w}(s, t)$ as:

$$
\bar{w}(s, t)=\frac{\int_{A_{C M D}} w(x, s, z, t) d x d z}{A_{C M D}}
$$

$$
\bar{w}(s, t)=\underline{\bar{w}}_{0}(s) \cdot \overline{\bar{w}}(t)
$$

with $\underline{\bar{w}}_{0}(s)$ being a time independent dimensionless function expressing the variation of water content along the longitudinal coordinate due to the variation of geometrical, hydraulic and lithological properties and to the effects of exposure to weather conditions, and $\overline{\bar{w}}(t)$ a space independent dimensional function, which accounts for the effect of climatic variations and aging on the ground water content.

Considering measurements of water content at $t_{\mathrm{k}}$, $w_{k}=w\left(x, s, z, t=t_{k}\right)$ can be expressed as the weighted average of the values of water content, $w_{i ; k}=w\left(x, s=s_{i}, z, t=t_{k}\right)$, recorded at $t=t_{\mathrm{k}}$ in the instrumented cross-sections (here the generic number $\mathrm{N}$ is used):

$w_{k}(x, s, z)=w\left(x, s, z, t=t_{k}\right)=\sum_{i=1}^{N} w_{i ; k}(x, z) \cdot \gamma_{i}(s)$

with $\gamma_{i}(s)$ being weight functions. The simplest choice for $\gamma_{i}(s)$ is to consider a linear variation between the values of water content measured at the $\mathrm{N}$ instrumented sections:

$$
\gamma_{i}(s)=\left\{\begin{array}{cccc}
1 & \text { for } & s=s_{i} & \forall i \in[1, . . N] \\
0 & \text { for } & s=s_{j \neq i} & \forall i, j \in[1, . . N] \\
1-\frac{\left(s-s_{i}\right)}{\left(s_{i+1}-s_{i}\right)} & \text { for } & s_{i} \leq s \leq s_{i+1} & \forall i \in[1, . . N] \\
1+\frac{\left(s-s_{i}\right)}{\left(s_{i-1}-s_{i}\right)} & \text { for } & s_{i-1} \leq s \leq s_{i} & \forall i \in[1, . . N]
\end{array}\right.
$$

The water content in the whole embankment is obtained by interpolating the values of water content measured by the geotechnical probes in the $\mathrm{N}$ sections where they are located:

$w_{k}(x, s, z)=w\left(x, s, z, t=t_{k}\right)=\sum_{i=1}^{N} w_{i ; k}(x, z) \cdot \gamma_{i}(s)$

Now the water content averaged over the whole embankment, $\overline{\bar{w}}(t)$, can be calculated from the water content measurements performed by the geotechnical probes in the monitored sections as:

$\overline{\bar{w}}(t)=\frac{\int_{0}^{L} \frac{\int_{A_{C M D}} \sum_{i=1}^{N} w\left(x, s=s_{i}, z, t\right) \cdot \gamma_{i}(s) d x d z}{A_{C M D}}}{L} d s$

and decompose it into two functions: 
$\overline{\bar{\sigma}}(t)$ and $\overline{\bar{w}}(t)$ are the functions considered to seek a correlation between electrical conductivity and water content. In doing so, the significant dependency exhibited by ground electrical conductivity on temperature [11] needs to be accounted for. According to the work of [12] on glacial tills, the relationship is linear, i.e. $\sigma=\sigma_{25}[C(T-25)+1]$ with $\sigma$ being the electrical conductivity measured at temperature $T$ and $\mathrm{C}=0.02$. The measured electrical conductivities need to be expressed in relation to a same reference temperature before being correlated to the water content measurements, so the following expression was employed:

$$
\sigma_{r e f}=\sigma \frac{1+C\left(T_{r e f}-25\right)}{1+C(T-25)}
$$

with $\sigma_{\text {ref }}$ being the value of electrical conductivity expressed in terms of the chosen reference temperature, $T_{\text {ref }}$. Here, $T_{\text {ref }}=15$ Celsius was chosen to minimise the amount of temperature compensation. Eq. (10) was used to calculate $\sigma_{\text {ref }}$ from the measured values of $\sigma$ and $T$.

In Figure 5, the water content measured at $t_{k}$, $\overline{\bar{w}}\left(t=t_{k}\right)$, is plotted against the electrical conductivity measured at the same time points, $\overline{\bar{\sigma}}_{r e f}\left(t=t_{k}\right)$. It emerges that the relationship between $\overline{\bar{w}}(t)$ and $\overline{\bar{\sigma}}_{r e f}(t)$ is well captured by a linear function so that:

$$
\overline{\bar{w}}(t)=m \overline{\bar{\sigma}}_{r e f}(t)+q
$$

with $m$ and $q$ determined by best fit. Substituting Eq. (11) into Eq. (12), we get:

$$
\overline{\bar{w}}(t)=m \overline{\bar{\sigma}}(t) \frac{1+C\left(T_{r e f}-25\right)}{1+C(T-25)}+q
$$

Eq. (13) relates the space average of the measured electrical conductivity to the space average water content.

To determine the water content in any generic point of the embankment, $w(x, s, z, t)$, the space independent dimensional function $\overline{\bar{w}}(t)$, must be multiplied by the normalised time independent function, $\underline{w}_{0}(x, s, z)$, so that:

$$
w(x, s, z, t)=\underline{w}_{0}(x, s, z) \cdot \overline{\bar{w}}(t)
$$

$\underline{w}_{0}(x, s, z)$ is the time average of the linear combination of the functions expressing the normalised water content measured at $t_{\mathrm{k}}$ in the instrumented sections, $w_{0}{ }_{i ; k}(x, z)$ :

$$
\begin{aligned}
& \underline{w}_{0}(x, s, z)=\underset{k}{\operatorname{average}}\left[\sum_{i=1}^{N} w_{0_{i ; k}}(x, z) \cdot \gamma_{i}(s)\right] \\
& \text { with } w_{0_{i ; k}}(x, z)=w_{0}\left(x, s=s_{i}, z, t=t_{k}\right)=\frac{w\left(x, s=s_{i}, z, t=t_{k}\right)}{\int_{A_{i}} w\left(x, s=s_{i}, z, t=t_{k}\right) d x d z} \\
& A_{i}
\end{aligned}
$$

The function $\underline{w}_{0}(x, s, z)$, is assumed to be:

$\underline{w}_{0}(x, s, z)=\bar{w}(s, t) \cdot \underline{\overline{\underline{\sigma}}}_{0}(s)=\underset{k}{\operatorname{average}}\left[\sum_{i=1}^{N} w_{0_{i, k}}(x, z) \cdot \gamma_{i}(s)\right] \underline{\bar{\sigma}}_{0}(s)$

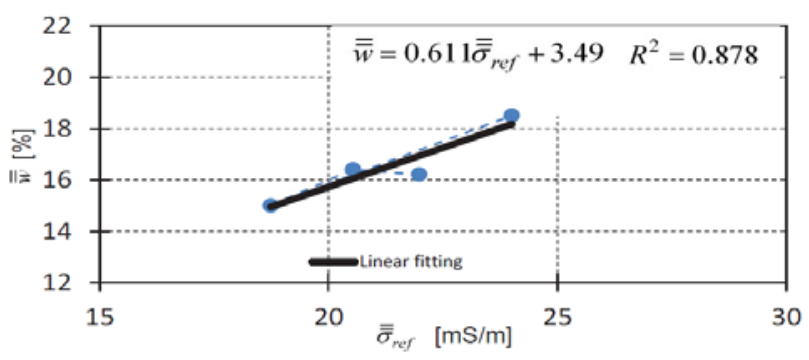

Fig. 5. Correlation between average electrical conductivity, $\overline{\bar{\sigma}}_{r e f}(t)$, and average water content $\overline{\bar{w}}(t)$ obtained for $d=0.2 \mathrm{~m}$ and $\mathrm{T}_{\text {ref }}=15^{\circ} \mathrm{C}$.

The advantage of employing geophysics is now apparent since unlike geotechnical measurements that can be taken only in a few discrete sections, it provides measurements which are continuous along the spatial coordinate $s$.

Substituting Eq. (13), and (16) into Eq. (14), the equation to convert electrical conductivity measurements (taken at the time $t_{C M D}$ ) into values of water content is obtained:

$$
\begin{aligned}
& w\left(x, s, z, t=t_{C M D}\right)=\left\{\underset{k}{\operatorname{average}}\left[\sum_{i=1}^{N} w_{0}\left(x, s=s_{i}, z, t=t_{k}\right) \cdot \gamma_{i}(s)\right] \cdot \overline{\underline{\sigma}}_{0}(s)\right\} . \\
& {\left[m \frac{\bar{\sigma}\left(s, t=t_{C M D}\right)}{\bar{\sigma}_{0}(s)} \frac{1+C\left(T_{r e f}-25\right)}{1+C(T-25)}+q\right]}
\end{aligned}
$$

Note that electrical conductivity measurements can take place when the geotechnical suite is no longer active (i.e. $\left.t_{C M D}>t_{k}\right)$.

\section{Validation}

To validate the proposed method, a comparison between the water content values determined from electrical 
conductivity measures according to the described procedure and water content measured in the laboratory from soil samples retrieved in situ is here illustrated. Contour lines of water contents in two randomly chosen cross-sections ( $s=2,182$ ) calculated using Eq. (17) from electrical conductivity measurements carried out on $17^{\text {th }}$ April 2014 (so for $t_{C M D}>t_{k}$ ) during a walk-over are plotted in Figure 6 . The values of the water content measured in the laboratory from in-situ retrieved samples are reported in the plots as triangles.

The values of water content converted from the conductivity measurements according to the proposed procedure show to be in very good agreement with the laboratory measures. Note that the conductivity measurements were taken at a time (17 $7^{\text {th }}$ April 2014) well beyond the 2 year period during which the calibration of the model was performed.

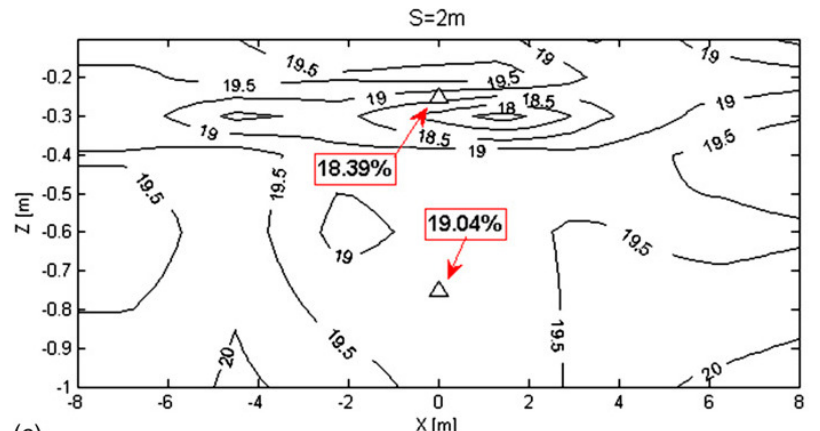

a)

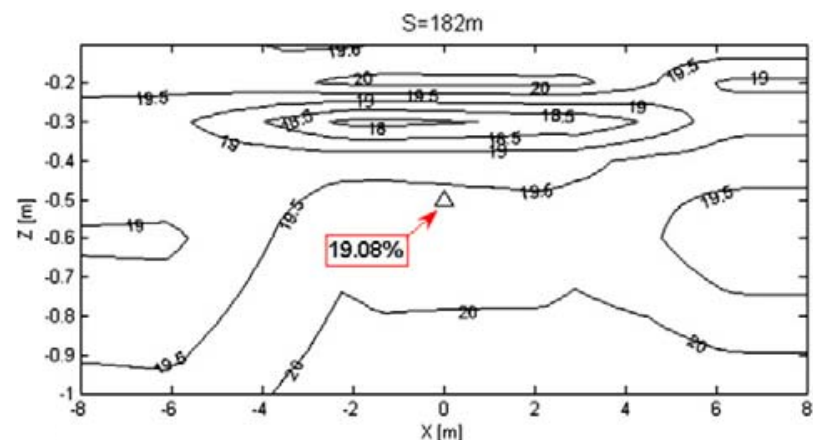

b)

Fig. 6. Values of water content estimated from electrical conductivity measurements (carried out on 17th April 2014) employing the proposed methodology. a) water content calculated at $\mathrm{s}=2 \mathrm{~m} ; \mathrm{b}$ ) water content calculated at $\mathrm{s}=182$. The triangles indicate values of water content measured in the laboratory from in-situ retrieved samples.

\section{Conclusions}

A methodology for the periodic monitoring of water content in embankments to be carried out by a noninvasive hand held geophysical device measuring electrical conductivity is proposed here. The methodology requires the use of geotechnical probes installed in at least two cross-sections of the embankment to calibrate the electrical conductivity water content relationship.

The values of water content converted from the conductivity measurements according to the proposed procedure were found to be in very good agreement with the laboratory measures even at times well beyond the 2 year period during which the calibration of the model was performed.

\section{Acknowledgements}

The financial contribution of EU H2020 RISE grant 'Hercules' (778360) is gratefully acknowledged.

\section{References}

1. J.A. Charles, Geotechnique 58, 541-570 (2008).

2. J. Perry, M. Pedley, M. Reid, Infrastructure embankment: condition appraisal and remedial treatment, CIRIA Report C550 (2001).

3. CIRIA, International Levee Handbook, CIRIA, London (UK), publication C731 (2013).

4. M. Morris, M. Dyer, P. Smith, J. Falkingham, J. Simms, Management of flood defences, Rep. FD2411, Defra/Environment Agency, London, (2007).

5. M. Dyer, S. Utili, M. Zielinski, Proc. ICE - Water Management, 162, 221-232 (2009).

6. W. Allsop, et al., Failure mechanisms for flood defence structures, EU FP7 FLOODSite task 4, Research Report: T04-06-01, (2007).

7. S. Utili, R. Castellanza, A. Galli, P. Sentenac, J. Geotech. Geoenviron. Engrg., 141, 04014111 (2015).

8. Gf Instruments, Short guide for electromagnetic conductivity survey, www.gfinstruments.cz (2011).

9. Zielinski M. Influence of desiccation fissuring on the stability of flood embankments. $\mathrm{PhD}$ thesis, University of Strathclyde, UK (2009).

10. Zielinski M., Sanchez M., Romero E., Sentenac P. Proc. ICE Geotech Engrg, 164, 139-148 (2011).

11. G.V. Keller, F.C. Frischknecht, Electrical Methods in Geophysical Prospecting, Pergamon Press, Oxford, (1966).

12. K. Hayley, L.R. Bentley, M. Gharibi, M. Nightingale, Geophysical Res. Letters, 34, L18402 (2007). 